\title{
CanMEDS scholars: A national survey on urology residents' attitudes towards research during training
}

Ogi Solaja, BSc; Thomas A.A. Skinner, MD; Thomas B. McGregor, MD; D. Robert Siemens, $\mathrm{MD}$

Department of Urology, Queen's University, Kingston, ON, Canada

Cite as: Can Urol Assoc J 2017 Dec. 22; Epub ahead of print. http://dx.doi.org/10.5489/cuaj.4927

Published online December 22, 2017

$* * *$

\section{Abstract}

Introduction: Participation in scholarly activity is an important tenet of residency training and is firmly entrenched in Canada since the introduction of CANMEDS roles by the Royal College of Physicians and Surgeons. As Canadian residency programs transition to competency-based training, it will remain important to understand how to best implement and encourage scholarly pursuits among resident trainees. The objective of this study was to understand the experiences, attitudes, and barriers that surgical residents face when pursuing research during their training. Methods: An anonymous, cross-sectional, self-report questionnaire was administered to chief residents of all English-speaking urology programs in Canada in 2015. Questions were open- and closed-ended, including an agreement score based on a five-point Likert scale. Questions addressed residents' involvement in and attitudes towards research, as well as their perceptions of the utility of research involvement during training. The residents were also asked about the support they received and potential areas to improve the attainment of this competency. Descriptive and correlative statistics were used to analyze the responses.

Results: There was a $100 \%$ overall response rate to the questionnaire. This study revealed that Canadian urology residents have a high rate of participation in scholarly work, with the vast majority (94\%) publishing at least one manuscript with a mean of four papers. Despite this, there appeared to be significant variation in the respondent's experiences, including protected time for research. Furthermore, many residents appeared unconvinced of the importance of research involvement, with only 51\% agreement that participation was important to their overall training. As well, a significant number of residents reported largely external, rather than internal, motivations for research participation, such as attaining a preferred fellowship (66\% agreement). While the majority of respondents felt (66\% agreement) that the scholar role was important in 
residency training, it would appear that significant barriers, including time and mentorship, limited the effectiveness of research participation to gain those competencies.

Conclusions: The results of this self-report survey outline the significant differences in attitudes and experience towards mandatory research as a component of scholarship in Canadian urology training programs. As postgraduate medical education evolves, particularly with the uptake of competency-based medical education, programs and residents will need to address the motives and barriers to better foster academic pursuits during urology training.

\section{Introduction}

Fears over dwindling numbers of physician-scientists are not new; James Wyngaarden, who later became the Director of the National Institutes of Health, is largely considered the first to ring the alarm, calling them an "endangered species" in $1979 .{ }^{1}$ Since then, the demonstrably declining and aging population of physician-scientists has become a topic of much discussion ${ }^{2,3}$. In 2016, a review of the literature by Kosik et al. identified over 1200 peer-reviewed publications that discuss salvaging the physician-scientist workforce. ${ }^{4}$ Suggested explanations of this trend include financial pressures of increased education costs, lower financial incentives, a changing philosophy, and inadequate exposure to research during medical training., ${ }^{5,6}$

Physician-scientists have long been valued for their combined clinical and research experience. This experience has reciprocal benefit, allowing for translation of knowledge at the bedside, as well as directing research with practical clinical questions. This is especially true in the modern evidence-based environment. In an effort to preserve this unique skill set, The Royal College of Physicians and Surgeons of Canada introduced the role of scholar to the early CanMEDS framework in 1993. This addition was insisted on by medical faculty, who felt that the original list of physician responsibilities didn't directly address the role of physicians as scientists. ${ }^{7}$ The persistence of this role has established scholarly work as a tenet of Western medicine, and set an expectation that medical trainees are to participate in some form of research during their training. Participation in research improves residents' ability to critically appraise literature, nurtures critical thinking, improves patient care, and influences career paths. ${ }^{8}$

Program directors generally agree on the importance of research, with many programs allotting research time for residents, and expecting or even requiring involvement in research projects. However, studies have shown discrepancies in the perception of both the quality and value of research training among residents in anesthesia, pediatric, physical medicine and rehabilitation, family medicine, psychiatry and internal medicine programs. ${ }^{8,9,10,11,12,13}$ Furthermore, it has been demonstrated that interest in research while high initially, wanes as residency progresses. ${ }^{14}$

The concern around the decline in the number and focus of clinician-scientists have led to several important initiatives over the last few decades including increasing early exposure to research at the medical school level, initiating medical scientist training programs, fostering research in sub-specialty fellowships and developing clinician-scientist/early career awards. 
There is still however much debate as to the effectiveness of these endeavors, particularly in the surgical specialties. To our knowledge, there is no information on how urology residents, or any other surgical residents for that matter, perceive research in residency. The objective of this study was to assess research attitudes of chief urology residents in Canada, to better understand their attitudes, experiences and barriers to their scholarly pursuits in training.

\section{Methods}

This prospective, cross-sectional study surveyed all PGY-5 residents from English-speaking Canadian programs $(n=35)$ while they attended a review course in 2015. Participation in the selfreported survey was voluntary and anonymous; no identifying information was collected. We obtained ethics approval from the Queen’s University Institutional Review Board, and an information package describing the motives, objectives, and confidentiality of the study were distributed with the surveys.

The survey consisted of 32 closed-ended questions and statements regarding research involvement. Of these, 27 could be answered with provided multiple choice options or a 5-point Likert agreeability scale. The rest required response with a free-written numerical value or short phrase. The first half of the questions assessed prior research experience, current research habits, and plans for future practice. The latter half assessed motives for participation in research, beliefs about its importance, and residents' impressions of their program's support. The survey concluded with one free-form open-ended question inviting the trainees to share their thoughts on how best to increase resident involvement in research. Questionnaire development resulted from an initial experience with a previous survey construction for similar attitudes for medical students. Students and educators involved in both under-graduate and post-graduate programs were asked to assess and modify the survey for clarity and was originally modified after a trial questionnaire exploring other intrinsic CanMEDS roles in 2009. In this present study we were able to compare and contrast some of the responses to those from the unpublished pilot survey in 2009 in an effort to assess stability of the responses over time.

Data was analyzed using descriptive statistics. Frequencies were tabulated and means with standard deviations used as appropriate to describe responses on the Likert scale. In cases where a participant responded with a range rather than a single value, the midpoint was used for analysis. That is, if a participant claimed to spend 2-4 hours on research per week, 3 hours was used in the analysis. For the purposes of reporting on questions using the 5-point Likert scale, the agreement responses 4 and 5 were grouped together, as were the disagreement responses 1 and 2 , unless otherwise stated. All other quantitative statistics utilized the full 5-point Likert scale. 


\section{Results}

In total, 35 final-year residents responded to the survey corresponding to a $100 \%$ response rate. When asked how much time on average they currently spent on research each month, the most common answer (11 of 35) was none; interestingly, the next most frequent (7 of 35) was 9 or 10 hours per month (Fig. 1). Overall, the median time spent on research was 4 hours per month. The likelihood of doing a fellowship was also bi-modal; 8 respondents (23\%) were not at all likely and 20 (57\%) were extremely likely to do a fellowship (Likert score mean +/- standard deviation (SD), 3.8+/-1.7 SD). Of the 26 participants that specified which fellowship they planned on pursuing, eleven were interested in MIS/endourology (42\%), seven in oncology (27\%), three in both infertility and reconstruction/female urology (12\%) and one in each of pediatric and transplant urology (4\%) (Fig. 2). When we inquired which setting they intended to practice in, 16 of 35 residents were inclined toward community practice, while 10 were interested in an academic practice; 9 were undecided.

With respect to research experience, the vast majority (94\%) of chief residents had published some manuscripts, with an average of 2 first authorships and 4 total publications each (Fig. 3). Most had participated in retrospective chart reviews and case reports/series (91\% and $57 \%$ respectively), while a smaller portion had been involved in basic science or prospective clinical trials (23\% each). A majority preferred involvement in clinical research (83\%) over basic science (3\%), with 6\% expressing equal preference and $9 \%$ expressing lack of interest in both.

When probed on their attitudes toward research, $66 \%$ agreed $(3.9+/-0.9 \mathrm{SD})$ that the 'scholar' role is an important CanMEDS component; 25\% responded neutrally and 9\% disagreed. Fifty-one percent agreed (mean 3.5 +/- 1.0 SD) that participation in research during residency was important to their overall training, and 46\% agreed (mean $3.3+/-1.2 \mathrm{SD}$ ) that it would be valuable when they eventually began practice in urology. Twenty-six of 35 residents (66\%) agreed (mean $3.4+/-1.3 \mathrm{SD}$ ) that they were doing research to improve their resume for fellowship, and $46 \%$ reported this as their main reason for participating, while $49 \%$ believed that involvement in research would increase their employability after residency. A majority (57\%) (mean 3.6 +/- 1.0 SD) felt extrinsic pressure to publish, and only 25\% (mean 3.1 +/- $0.9 \mathrm{SD}$ ) felt a personal obligation to advance the practice of urology through research.

When questioned about their programs, 14 residents (40\%) reported that they were allotted no official time for research, with another 14 reporting up to two months and 7 (20\%) claiming to have over 2 months of dedicated research time. Overall, only 34\% (mean $2.1+/-1.1$ $\mathrm{SD}$ ) of residents agreed that they had enough time for research in residency; in fact, "strongly disagree" was the most common response to that statement (12 of 35). Conversely, participants emphatically agreed (74\%) (mean 4.0 +/- 1.2 SD) that they would be more inclined to do research if there was more time specifically set aside for it (Fig. 4). Given an opportunity to suggest what could be done to increase interest in research, virtually all comments mentioned 
more protected research time, help in generating ideas for projects, and more supportive mentorship from staff.

Interestingly, there was no strong correlation between reported time allotted for research in residency and likeliness to practice in an academic setting (Spearman's $r=0.244, p=0.79$ ). However, there was a positive correlation between likeliness to pursue an academic practice and number of publications (Spearman's $r=0.407, p=0.025$ ) and there was a trend to an association of pursuit of an academic practice and personal time spent on research endeavors (Spearman's $\mathrm{r}=0.259, \mathrm{p}=0.067$ ). The number of first authorships was strongly correlated to having a research mentor or role model to emulate (Spearman's $r=0.461, \mathrm{p}=0.03$ ). Surprisingly, there was no correlation between the amount of research time allotted by the program and the number of papers published by each resident (Spearman's $r=0.03$ ). In fact, there was no correlation between the time allotted for research and the time spent on research (Spearman's $r=-0.05$ ). Although the difference was not statistically significant, residents intending to go into oncology had published more studies on average than those intending to do MIS/endourology (7.33 $\pm \mathrm{SD}$ 2.2 vs $4.5 \pm$ SD 5 respectively, $\mathrm{t}=-1.6, \mathrm{p}=0.12$ ).

An unpublished 2009 survey of chief urology residents administered in a similar manner included several of the same questions, allowing for direct comparison of responses over time. There was no statistically significant difference between the 2009 and 2015 cohort in regards to the agreement of the importance of the scholar role (69\% - $3.9+/-0.9$ SD vs. $66 \%-3.9+/-0.9$ SD), importance of research in residency ( $50 \%-3.3+/-1.4$ SD vs. $51 \%-3.5+/-1.0$ SD), number of papers published (4.5 vs. 4 ), perception of adequate time for research (19\% - $2.5+/$ 1.3 SD vs. $24 \%-2.1+/-1.1 \mathrm{SD})$, and inclination to do research if allotted more time $(72 \%-2.5$ +/- 1.3 SD vs. $74 \%(4.0+/-1.2$ SD).

\section{Discussion}

The role of physician as scientist has arguably never been more essential than in today's evidence-driven, information-laden practice environment. Various educational initiatives, including the CanMEDS framework, exalt the importance of scholarly pursuit, yet fears grow over waning interest. Although some studies have characterised resident publishing patterns and demonstrated the benefits of research in residency, this is - to the best of our knowledge - the first study to examine urology residents' motivations for and attitudes towards research. ${ }^{15,16,17,18}$ These results demonstrate significant ambivalence among residents, and identify possible ways to improve interest.

The results of this self-report survey showed that 94\% of Canadian urology residents had published manuscripts; publishing or submitting an average of four papers each, including two as first author, comparing favourably to reports by US counterparts. ${ }^{16,17,18}$ A 2013 study by Andrews et al. assessed the productivity of urology residents across Canada, surveying 42 residents from 10 programs and across all years; they found that $83 \%$ of respondents were involved in research at the time, with an average of $1.25 \pm 0.37$ publications. ${ }^{15}$ Conversely, we 
found that 24 of 35 (69\%) final-year residents were actively involved in research at the time of our survey, suggesting that the brunt of publications came later in residency, but also likely that active involvement in research wanes as the end of residency approaches.

The majority of respondents felt (66\% agreement) that the scholar role was important in residency training. These figures are encouraging in light of evidence demonstrating that academic productivity in residency was associated with greater pursuit of fellowship training, tendency to academic practice, and productivity in future careers. ${ }^{16,17,19}$ As a whole, however, the participants' responses suggest a personal ambivalence around the importance of research during training and appear extrinsically — rather than intrinsically_motivated to participate. While twothirds of chief residents agreed that Scholar was an important CanMEDS role, only half thought that participating in research was important to their training and fewer still believed that it would be valuable when they began their practice. Perhaps this result can be explained by the timing of the survey and the commonly expressed preference for community over academic practice. These trainees may be unconvinced of the utility of research skills outside of academic centers, or may be aware but uninterested in participating when asked this question at the very end of their surgical training. The generally favourable attitude towards the CanMEDS scholar role indicates that these residents agree with the CanMEDS tenets of scholarly pursuit as they pertain to evidence-based practice, ongoing learning, and critical appraisal, but not necessarily to creating and disseminating knowledge in the form of published research.

Survey questions attempting to examine motives for participating in research endeavors suggested that trainees may be compelled to do research to improve their marketability, rather than contribute to the specialty per se; $46 \%$ responded that their main reason for doing research was to increase their chances of obtaining a preferred fellowship. This figure is congruent with the proportion of respondents that are extremely likely to do a fellowship (57\%), and presumably represents the stress of vying for competitive academic fellowship positions. This pressure is certainly compounded by competing clinical responsibilities, as two-thirds of residents expressed that they did not have enough time to do research. As more than half of residents reported feeling extrinsic pressure to attain research success, one could interpret these findings somewhat as a failure of our programs to facilitate and encourage research interest in these highly competent surgical residents. Not all blame should rest with our programs, however. It is equally fair to posit that system-level barriers stifle research during surgical training; a high burden of clinical responsibilities with sub-optimal University or hospital support and funding are potentially insurmountable challenges in today's environment. Several surgical residency programs have implemented dedicated research streams that integrate a year or two of full-time research into their curriculums. These programs present significant administrative and coordinative challenges, and may not be feasible for small programs that only support one or two residents per year. Additionally, with the recent shift to competency-based medical education one must speculate whether residents will find even less time to conduct research unless specifically 
addressed in the competency framework. With this said, given the taxing schedules of our surgical residents leaving little dedicated time for intensive research activities, our study still highlights significant engagement in the Scholar role and, despite the obstacles, speaks volumes about their commitment to academic pursuits in training.

This sentiment is further evidenced by the fact that $74 \%$ of residents would be more inclined to do research if there was more time set aside for it. Yang et al. surveyed graduating residents from the top 50 urology programs in the US and found that publication output greatly correlated with increasing dedicated research time. ${ }^{16}$ Hellenthal et al., who similarly surveyed chief residents in the US and Canada, also found that the number of months allotted for research time was a significant predictor of resident manuscript prodctivity. ${ }^{17}$ Surprisingly, we found no strong correlation between allotted research time (self-reported) and academic productivity. This may be because the time allotted (mean $4.3 \pm 5.5 \mathrm{SD}$ weeks) is insufficient to cultivate additional publications. In comparison, Yang et al. found that $65 \%$ of the residents they surveyed had at least 3 months of protected research time, with $37 \%$ reporting a full year. ${ }^{16}$ This further underscores the productivity of Canadian urology residents, who reported an average of 4.4 weeks of research time set aside by their programs. What appears more surprising is the lack of correlation of the survey questions examining allotted research time and self-reported time spent on research. This may be partially explained by some recall bias when asked to report their current time commitment to research, in the last months of their final training year, and may not be representative of the time they spent on research throughout their training. Interestingly, we also found that first authorship was strongly correlated with the degree of mentorship and role modelling residents experienced. This is an influence that has previously been identified in pediatric and emergency residents. ${ }^{14,20}$ Other predictive factors identified in our search of the literature include the number of residents in the program and formal research training in the form of a Masters or $\mathrm{PhD} .{ }^{18,21}$

Comparison of our results to those obtained from a similar survey in 2009 reveal that research habits, motivations, and attitudes are remarkably stable over time. This lends validity to our findings; it emphasizes the academic merits of urology residents, but also further underscores one of their stated barriers to fulsome participation - the lack of time to devote to it.One of the shortcomings of this study includes a small sample size of 35 that included a single cohort; tendencies likely vary year-to-year although the stability of the responses from 2009 to 2015 were remarkable. Another is the timing of the survey, which was administered shortly before the residents were to write their Canadian certification exams. This is especially influential given the self-reporting nature of the survey and accompanying recall bias. Despite these limitations, the $100 \%$ response rate provides a comprehensive look into the experiences, attitudes and motivations of Canadian urology residents as they pertain to research. Further queries of residents in different years of training as well as those involved with the implementation and assessment of competency-based curriculum in urology to collect more objective data—such as 
time allotted for research, available resources, and productivity—would be valuable to confirm the veracity of our findings and aid in the development of curriculum/experiences within our programs to best attain competencies in the Scholar role.

\section{Conclusion}

This study provides insight into research attitudes of Canadian urology residents. Given the importance of scholarly activity and awareness of research methods in today's evidence-based practice environment, programs may use some of these findings to improve residents' experiences and research productivity. As Canadian residency training programs transition to a competency-based design, they may be able to use this information to inform decisions on how best to implement and encourage scholarly pursuits among residents. 


\section{References}

1. Wyngaarden JB. The Clinical Investigator as an Endangered Species. $N$ Engl J Med. 1979;301(23):1254-1259. doi:10.1056/NEJM197912063012303.

2. Ley TJ, Rosenberg LE. The physician-scientist career pipeline in 2005: build it, and they will come. JAMA. 2005;294(11):1343-1351. doi:10.1001/jama.294.11.1343.

3. Solomon SS, Tom SC, Pichert J, Wasserman D, Powers AC. Impact of Medical Student Research in the Development of Physician-Scientists. J Investig Med. 2003;51(3):149156. doi:10.1136/jim-51-03-17.

4. Kosik RO, Tran DT, Fan AP-C, et al. Physician Scientist Training in the United States. Eval Health Prof. 2016;39(1):3-20. doi:10.1177/0163278714527290.

5. Lloyd T, Phillips BR, Aber RC. Factors that influence doctors' participation in clinical research. Med Educ. 2004;38(8):848-851. doi:10.1111/j.1365-2929.2004.01895.x.

6. Schafer AI. The vanishing physician-scientist? Transl Res. 2010;155(1):1-2. doi:10.1016/j.trsl.2009.09.006.

7. Whitehead CR, Austin Z, Hodges BD. Flower power: the armoured expert in the CanMEDS competency framework? Adv Heal Sci Educ. 2011;16(5):681-694. doi:10.1007/s10459-011-9277-4.

8. Abramson EL, Naifeh MM, Stevenson MD, et al. Research training among pediatric residency programs: a national assessment. Acad Med. 2014;89(12):1674-1680. doi:10.1097/ACM.0000000000000404.

9. Gill S, Levin A, Djurdjev O, Yoshida EM. Obstacles to residents' conducting research and predictors of publication. Acad Med. 2001;76(5):477. http://www.ncbi.nlm.nih.gov/pubmed/11346527. Accessed March 30, 2017.

10. Buschbacher R, Braddom RL. Resident versus program director perceptions about PM\&amp;R research training. Am J Phys Med Rehabil. 74(2):90-100. http://www.ncbi.nlm.nih.gov/pubmed/7710735. Accessed March 30, 2017.

11. Silcox LC, Ashbury TL, VanDenKerkhof EG, Milne B. Residents’ and Program Directors’ Attitudes Toward Research During Anesthesiology Training: A Canadian Perspective. Anesth Analg. 2006;102(3):859-864. doi:10.1213/01.ane.0000194874.28870.fd.

12. Crawford P, Seehusen D. Scholarly activity in family medicine residency programs: a national survey. Fam Med. 2011;43(5):311-317. http://www.ncbi.nlm.nih.gov/pubmed/21557099. Accessed March 30, 2017.

13. MacMaster FP, Cohen J, Waheed W, et al. The psychiatry resident research experience. BMC Res Notes. 2016;9(1):486. doi:10.1186/s13104-016-2290-1.

14. Neacy K, Stern SA, Kim HM, Dronen SC. Resident perception of academic skills training and impact on academic career choice. Acad Emerg Med. 2000;7(12):1408-1415. http://www.ncbi.nlm.nih.gov/pubmed/11099432. Accessed March 30, 2017.

15. Andrews JM, Abdolell M, Norman RW. Canadian urology resident scholarly performance. Can Urol Assoc J. 2013;7(5-6):402. doi:10.5489/cuaj.1348.

16. Yang G, Zaid UB, Erickson BA, Blaschko SD, Carroll PR, Breyer BN. Urology resident publication output and its relationship to future academic achievement. J Urol. 2011;185(2):642-646. doi:10.1016/j.juro.2010.09.097. 
17. Hellenthal NJ, Ramírez ML, Yap SA, Kurzrock EA. Manuscript Publication by Urology Residents and Predictive Factors. J Urol. 2009;181(1):281-287. doi:10.1016/j.juro.2008.09.022.

18. Finkelstein JB, Van Batavia JP, Rosoff JS. The Difference a Year Can Make: Academic Productivity of Residents in 5 vs 6-Year Urology Programs. Urology. 2015;86(2):220223. doi:10.1016/j.urology.2015.03.057.

19. Macknin JB, Brown A, Marcus RE. Does Research Participation Make a Difference in Residency Training? Clin Orthop Relat Res. 2014;472(1):370-376. doi:10.1007/s11999013-3233-y.

20. Ullrich N, Botelho CA, Hibberd P, Bernstein HH. Research during pediatric residency: predictors and resident-determined influences. Acad Med. 2003;78(12):1253-1258. http://www.ncbi.nlm.nih.gov/pubmed/14660429. Accessed April 4, 2017.

21. Merani S, Switzer N, Kayssi A, Blitz M, Ahmed N, Shapiro AMJ. Research Productivity of Residents and Surgeons With Formal Research Training. J Surg Educ. 2014;71(6):865870. doi:10.1016/j.jsurg.2014.05.007. 


\section{Figures and Tables}

Fig. 1. Number of hours, on average, currently spent on research per month. Overall, the median time spent on research was four hours per month. As one might expect, time spent on research appeared to correlate with likeliness of academic practice.

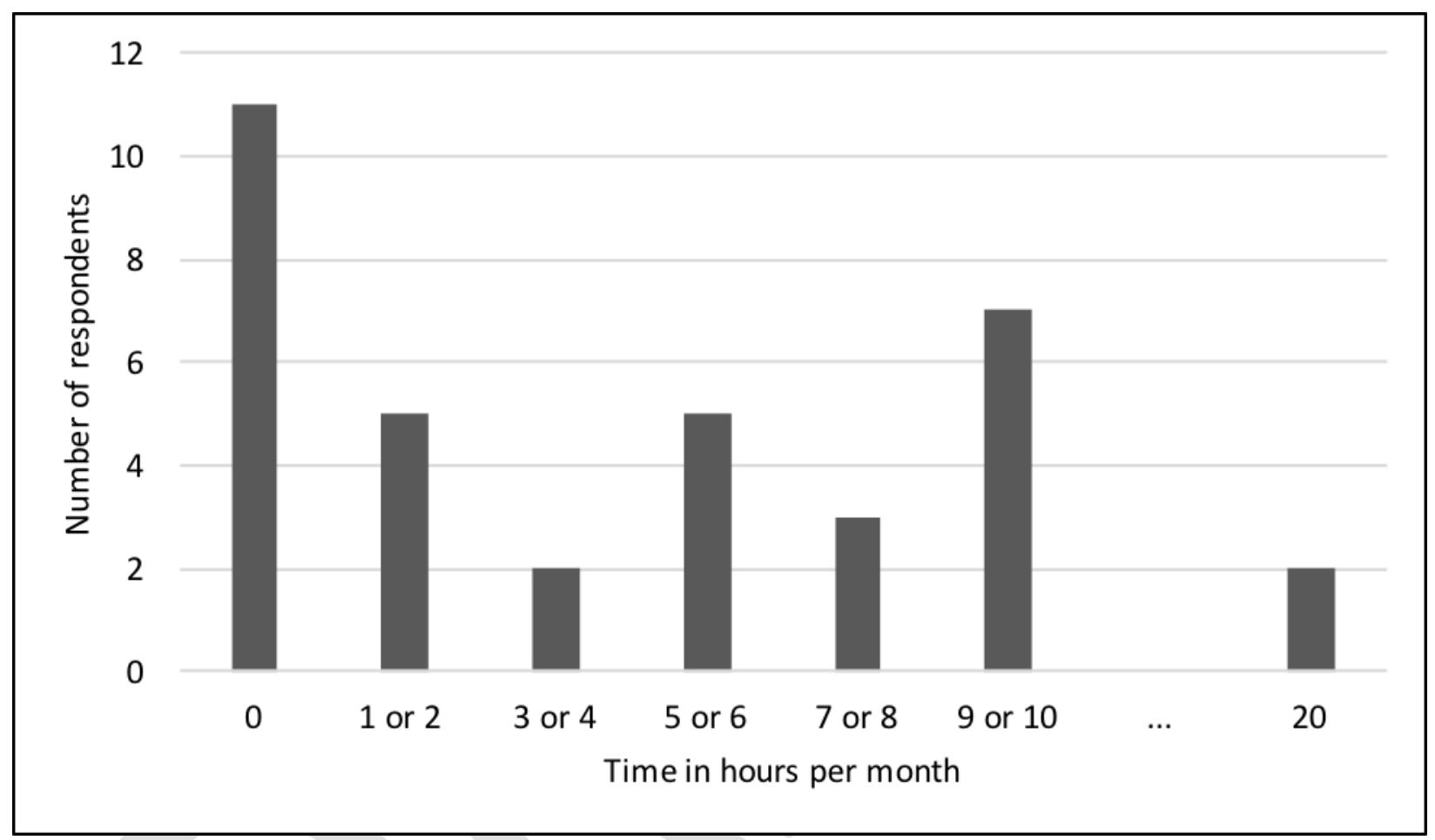


Fig. 2. Breakdown of fellowship inclinations among residents. Twenty-six of 35 residents responded; the rest, presumably, did not plan on doing a fellowship.

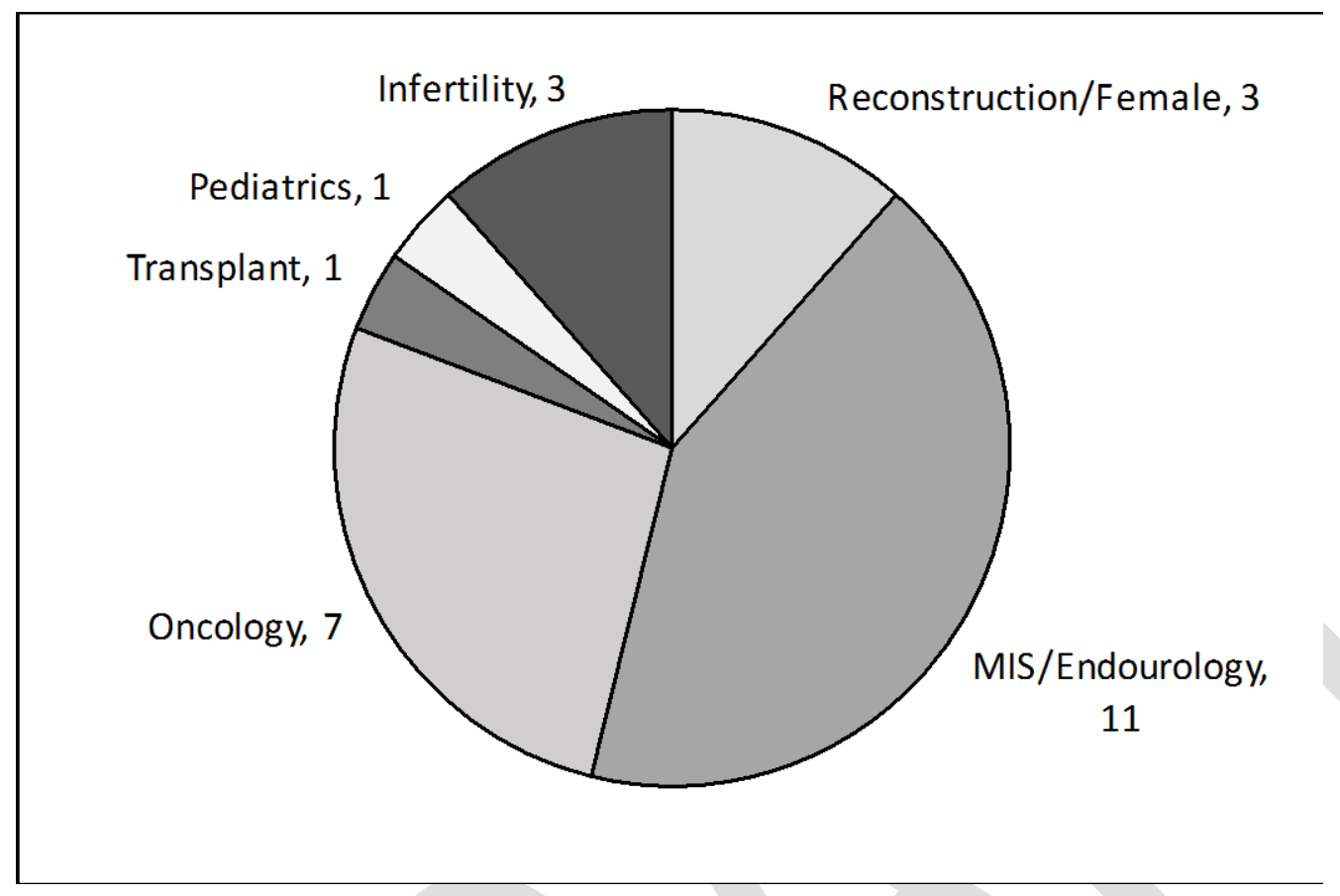


Fig. 3. Number of publications reported by urology residents in their final year. These include all papers published or submitted with the resident's name. The vast majority (94\%) of chief residents had published, with an average of two first authorships and four total publications each. Most had participated in retrospective chart reviews and case reports/series ( $91 \%$ and 57\%, respectively).

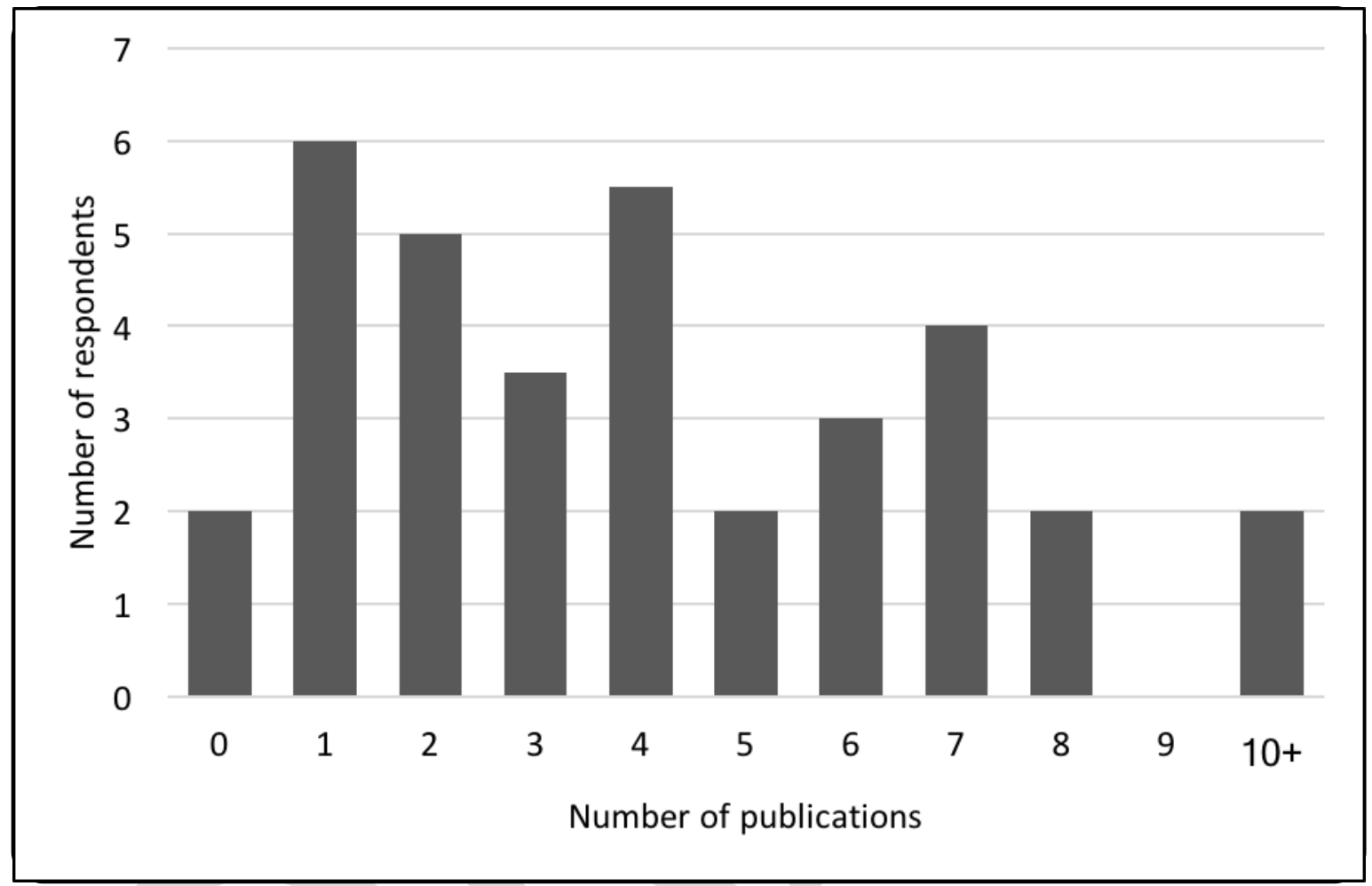


Fig. 4. Resident attitudes toward research. Thirty-five chief residents responded to a series of statements using a five-point Likert scale. In general, it appears that residents appear unconvinced of the importance of research and are extrinsically motivated to participate. They do, however, identify a paucity of allocated research time as a reversible barrier to scholarly pursuits.

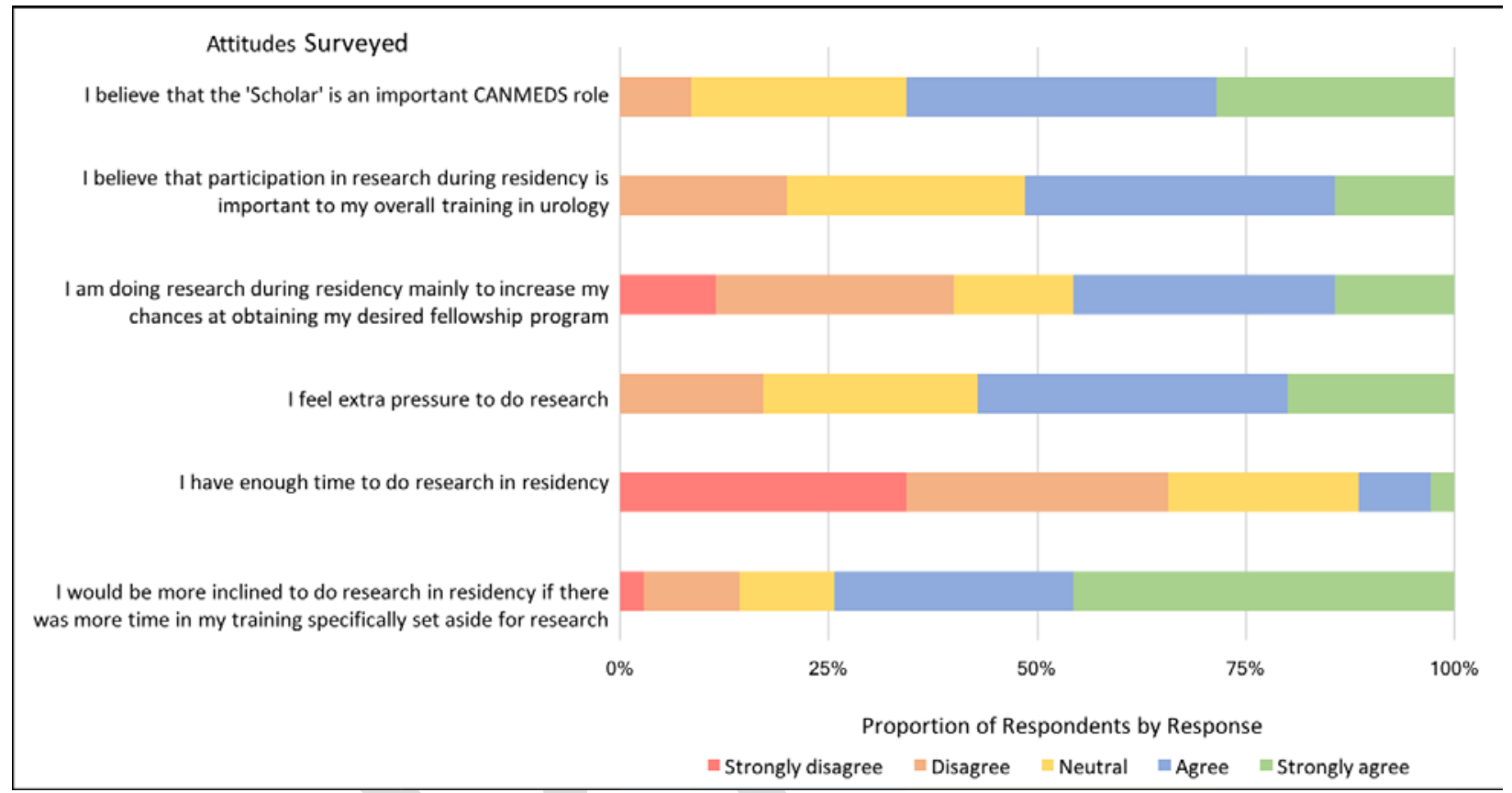

